\title{
A Molecular Dynamics Study on the Constraint Conditions of the Particle Growth Process in Laser Synthesis of Nanopowders
}

\author{
Shiwei Zhang,, Jun Liu, ${ }^{2}$ Zhijun Zhang, ${ }^{1}$ and Wenhui Zhang1 \\ ${ }^{1}$ School of Mechanical Engineering and Automation, Northeastern University, Shenyang 110004, China \\ ${ }^{2}$ Normal School, Shenyang University, Shenyang 110015, China
}

Correspondence should be addressed to Shiwei Zhang, shwzhang@mail.neu.edu.cn

Received 30 May 2012; Revised 21 August 2012; Accepted 27 August 2012

Academic Editor: Sinnanadar Ramasamy

Copyright ( 2012 Shiwei Zhang et al. This is an open access article distributed under the Creative Commons Attribution License, which permits unrestricted use, distribution, and reproduction in any medium, provided the original work is properly cited.

\begin{abstract}
Laser-induced chemical vapor deposition (LICVD) is a nanopowder synthesis method in which the nanoparticles of a synthetic product undergo nucleation, growth, and agglomeration. The growth process is crucial because it directly determines the growth rate and final size of nanoparticles. In this paper, the nanoparticle growth process is analyzed through a molecular dynamics study, and the process is divided into five steps. In addition, this study explains the microscopic heat and mass transfer processes that occur in the surrounding space and on the particulate surface. Three constraint conditions that may restrict the growth process, namely, transfer constraint, surface constraint, and temperature constraint conditions, are proposed and modeled. To calculate the final diameter and the nanoparticle growth rate, formulae for the constraint conditions are developed. The behavior of four gases in the particulate growth zone is discussed in detail.
\end{abstract}

\section{Introduction}

Laser-induced chemical vapor deposition (LICVD) is a nanopowder synthesis method which produces nanopowders that have excellent properties such as high purity, small particle size, narrow particle size distribution, good surface cleanliness, and the absence of hard agglomerates [1-5]. During LICVD synthesis of nanopowders, the gaseous precursor is injected into the reaction chamber and pyrolyzed by using a laser beam, and the vapor molecules of the desired solid product as well as gaseous byproducts are generated in the region of the laser beam. The molecular combination causes the initial cores to appear in the vapor at high temperature and supersaturation. The partial pressure and temperature of the vapor decrease along with the gas flow as a result of the diffusion loss and condensation on the core surface. The condensation causes the cores to continue absorbing the colliding vapor molecules and grow into nanoparticles. The nanoparticles stop growing when the vapor pressure is equal to the balance pressure of the particle surface. The particles become visible nanopowder as a result of agglomeration and the flow of the residual exhausted gas towards the collector.
During LICVD synthesis, the nanoparticles undergo nucleation (core formation), growth, and agglomeration. The growth process plays a key role because it determines the final particle size. Several factors affect the growth process. These factors are the constraint conditions of the growth process [6-9]. This paper details the mechanism of the particle growth process on a molecular scale, and the heat and mass transfers in the process are analyzed as constraint conditions through a molecular dynamics study.

\section{Growth Process Analysis}

An analysis of the growth process of the nanoparticles at the molecular scale includes the following steps.

(1) Transfer of gas phase particles from the surrounding space to the surface of the cores. The gas phase particles injected into the core surface include the vapor molecules of the desired solid product, molecules of various residual gaseous reaction products, gaseous precursor molecules that did not decompose, argon 
molecules for beam protection, and active particles of intermediate reaction products.

(2) Adsorption of gas phase particles on the nanoparticle surface. When the gas phase particles of various components appear at the surface, most particles will be physically adsorbed on the surface because of the intermolecular van der Waals force and will temporarily remain on the surface. A small number of these particles will be rejected directly and reflected elastically due to poor collision. The active particles of the intermediate products are more prone to adsorption.

(3) Phase change in which the product vapor molecules in the adsorbent state change into the solid phase state through condensation or a chemical reaction. The particles of the desired solid products on the adsorption surface, whether they are vapor molecules or active intermediate product fragments, tend to combine because their chemical composition and structure are basically the same as the cores. This process involves an interatomic valence bond and chemical adsorption, and the latent phase change heat is released. The adsorbed particles then become part of the nanoparticles.

(4) Desorption of other gas particles on the nanoparticle surface. Various gas particles adsorbed physically on the nanoparticle surface achieve temperature balance with the surface. These particles eventually break away from the surface as a result of the impact of subsequent incident particles, the vibration and collision of the solid surface molecules, or surface thermal evaporation. Some vapor molecules of the desired solid products also leave the surface, and the amount of evaporation depends on the equilibrium vapor pressure of the particulate surface temperature and its radius.

(5) Transfer of other gas particles after desorption. The desorbed gas particles must return to the surrounding space after leaving the particulate surface. Through convection and diffusion, the particles should then spread in a timely manner beyond the space range in which the nanoparticles directly exchange mass and heat with the surrounding environment. This process provides a suitable environment in which nanoparticles can grow sequentially.

Particle growth rate is constrained by the slowest step of the above processes. Steps (1) and (5) refer to the mass transfer of the gas phase material in the surrounding space that provides the gas phase transfer constraint condition for nanoparticle growth. Steps (2), (3), and (4) occur on the particulate surface and refer to the physical or chemical dynamic processes of surface adsorption as well as heat exchange between the nanoparticles and the surrounding environment. These steps provide the surface constraint and temperature constraint conditions for nanoparticle growth.

\section{Transfer Constraint Condition}

The molecular mass transfer process occurs around each nanoparticle within the space of several molecular mean free paths. The process takes place either before the molecules collide or after they leave the surface and will directly affect the environment for nanoparticle growth. Thus, if the gas compositions gathered around the nanoparticles could not exchange heat and mass with the farther surrounding space in a timely manner, they would consist mainly of the exhausted gas that returned from the particulate surface and would achieve componential and temperature balance with the particulate surface. As a result, the nanoparticles stop growing. Therefore, the molecular mass transfer process is a constraint condition for the nanoparticle growth process.

In the equation used for the molecular transfer constraint condition of particle growth, $V_{f 0}\left(\mathrm{~m}^{3}\right)$ is the volume of the nucleation region in the reaction flame (usually considered the laser focus zone) and $J\left(\mathrm{~m}^{-3} \mathrm{~s}^{-1}\right)$ is the nucleation rate. The nucleation rate of the critical condensing cores in the supersaturated vapor was derived by Zeldovich [10] as

$$
J=n_{2}^{2} V_{s 0} \sqrt{\frac{2 \sigma}{\pi m_{2}}} \cdot \exp \left(-\frac{4 \pi \sigma r_{c}^{2}}{3 k T}\right) \quad\left(\mathrm{m}^{-3} \mathrm{~s}^{-1}\right),
$$

where $n_{2}\left(\mathrm{~m}^{-3}\right)$ is the molecular number density of the product vapor, $m_{2}(\mathrm{~kg})$ is the mass of a single molecule, $V_{s 0}\left(\mathrm{~m}^{3}\right)$ is the volume occupied by each molecule in the solid nanoparticles, $r_{c}(\mathrm{~m})$ is the critical radius of the nanoparticles, $k=1.38 \mathrm{~J} \mathrm{~K}^{-1}$ is the Boltzmann constant, $T(\mathrm{~K})$ is the temperature, and $\sigma\left(\mathrm{J} \mathrm{m}^{-2}\right)$ is the unit area surface energy of solid nanoparticles. $\sigma$ has lower measured values on the nanoparticle surface than on a macroscopically flat solid surface as a result of the size effect of nanoparticles.

Therefore, the number of new initial cores generated in the nucleation zone in unit time is $N=J V_{f 0}\left(\mathrm{~s}^{-1}\right)$. The volume of the newly generated gas (product vapor and byproduct gas) in the nucleation zone is $U_{f}\left(\mathrm{~m}^{3} \mathrm{~s}^{-1}\right)$, and the volume density of the new condensation nucleus in the newborn gas is $n_{h}=N / U_{f}\left(\mathrm{~m}^{-3}\right)$. Thus, each condensation core may, on average, have a gas volume given by

$$
V_{h}=\frac{U_{f}}{V_{f 0} V_{s 0}} \cdot \frac{1}{n_{2}^{2}} \sqrt{\frac{\pi m_{2}}{2 \sigma}} \cdot \exp \left(\frac{4 \pi \sigma r_{c}^{2}}{3 k T}\right) \quad\left(\mathrm{m}^{3}\right)
$$

The molecular transfer constraint condition of particle growth can also be explained as follows: during the particle growth period, if all the vapor molecules within volume $V_{h}$ around the particle can reach the particle surface through diffusion migration (the molecular migration distance is about $\sqrt[3]{V_{h}}$ ) then the molecular transfer process can promote nanoparticle growth.

If the average length of a particle growth zone (usually the zone of the laser beam) is $L_{f}$ and the average velocity of vapor molecules is $u_{f}$, then the average time of the particles which pass through the growth zone is $\tau_{f}=L_{f} / u_{f}$. According to the theory of gas dynamics, a gas molecule that travels at time $\tau_{f}$ and frequently collides with other molecules that undergo three-dimensional random walks (Brownian 
motion) can move to the distance as long as its mean square root displacement [11] is

$$
\begin{gathered}
x_{\tau}=\left(\overline{x^{2}}\right)^{1 / 2}=\left(\frac{2}{3} \overline{\lambda_{2}} \overline{c_{2}} \tau_{f}\right)^{1 / 2}, \\
\overline{\lambda_{2}}=\left(\sum \pi \sqrt{1+\frac{m_{2}}{m_{j}}} \cdot\left(\frac{\sigma_{2}+\sigma_{j}}{2}\right)^{1 / 2} n_{j}\right)^{-1},
\end{gathered}
$$

where $\overline{c_{2}}=\sqrt{8 k T / \pi m_{2}}$ is the average velocity of vapor molecules, $\overline{\lambda_{2}}$ is the mean free path of vapor molecules in the gas mixture [11], and $\sigma_{j}(\mathrm{~m})$ is the effective diameter of various gas molecules.

Thus, the molecular transfer constraint condition of the nanoparticle growth process is given as

$$
x_{\tau} \geq \sqrt[3]{V_{h}} .
$$

Further results can be inferred based on (2). If all the product vapor is condensed, each nanoparticle that grows from an initial core will, on average, occupy the product vapor mass:

$$
M_{h}=\frac{U_{f} m_{2}}{V_{f 0} V_{s 0} n_{2}} \cdot \sqrt{\frac{\pi m_{2}}{2 \sigma}} \cdot \exp \left(\frac{4 \pi \sigma r_{c}^{2}}{3 k T}\right) \quad(\mathrm{kg}) .
$$

Each particle contains the number of product molecules given by

$$
g=\frac{M_{h}}{m_{2}}
$$

and the final particle diameter is

$$
D=\left(\frac{3 V_{s 0} \cdot M_{h}}{4 \pi m_{2}}\right)^{1 / 3} \quad(\mathrm{~m}) .
$$

Thus, a higher initial density of the condensation cores in the nucleation zone yields smaller nanoparticles.

\section{Surface Constraint Condition}

During nanoparticle growth, the various incident gaseous particles undergo a series of processes on the particle surface, including adsorption, residence, desorption, and phase change, which are all part of the surface action process. If one of these processes occurs slowly, the particle growth rate will be restricted and the process becomes a surface constraint condition.

The formation time of either the intermolecular van der Waals force or the interatomic valence bond force is sufficiently short (within $10^{-12} \mathrm{~s}$ ). Adsorption, desorption, and phase change occur over a short period and will not delay nanoparticle growth. Nanoparticle growth can be greatly affected by a large amount of gas molecules that are absorbed on the nanoparticle surface and remain there for a relatively long time, which prevents subsequent vapor molecules from reaching the nanoparticle surface. The calculation of the particle growth rate determined by using the surface constraint condition is shown in what folows.
According to the desorption dynamics of gas molecules, the average residence time of gas molecules on the surface can calculated by using the Frenkel-Arrhenius formula $[11,12]$

$$
\tau=\tau_{0} \exp \left(\frac{E_{d}}{k T}\right) \quad(\mathrm{s}),
$$

where $\tau_{0}(\mathrm{~s})$ is the vibration period of adsorbed molecules on the surface and is approximately equal to the vibration period of atoms or molecules on the surface, which ranges from about $10^{-12} \mathrm{~s}$ to $10^{-14} \mathrm{~s}$, and $E_{d}(\mathrm{~J})$ is the desorption activation energy for each gas molecule. The measured values of the activation energy on the nanoparticle surface are much lower than those on the macroscopically flat solid surface because of the size effect of nanoparticles. Moreover, the gas temperature in the particle growth zone is high. Thus, the gas molecules on the particulate surface have a short average residence time.

On the per unit area of the particulate surface, the number of adsorption sites is denoted as $\phi_{m}\left(\mathrm{~m}^{-2}\right)$, that is, as many as $\phi_{m}$ incident particles can be adsorbed. Among the incident particles, only those that reach the empty sites can be adsorbed on the surface. Within a certain period of the nanoparticle growth process, the surface coverage of gas molecules is denoted as $\theta$; that is, the number of adsorbed gas molecules on the per unit area particulate surface is, on average, equal to $\phi=\theta \cdot \phi_{m}$. Based on dynamic equilibrium, after residence time $\tau$, these adsorbed molecules $\phi$ will be desorbed and then escape from the surface. At the same time, new molecules $\phi$ adsorb at the empty sites of the surface, and these molecules are equal to the number of incident molecules at $1-\theta$ part of the per unit area of the particulate surface in $\tau$ time. Therefore,

$$
\phi=\theta \cdot \phi_{m}=\frac{p}{\sqrt{2 \pi m k T}} \cdot \tau \cdot(1-\theta) .
$$

A stable surface coverage of gas molecules can then be obtained by

$$
\theta=\frac{b p \tau}{1+b p \tau}
$$

where $b=1 /\left(\phi_{m} \sqrt{2 \pi m k T}\right)$ and $p, m$ are the total pressure and reduced molecular mass of various gas components, respectively.

When the particulate surface coverage is $\theta$, the mass growth rate of nanoparticles determined by the surface adsorption capacity is

$$
\begin{aligned}
\frac{d M}{d t} & =4 \pi r^{2}(1-\theta) \frac{p_{2}}{\sqrt{2 \pi m k T}} \cdot m_{2} \\
& =\frac{4 \pi r^{2} \varphi_{m} \sqrt{m_{2} m} \cdot p_{2}}{\varphi_{m} \sqrt{2 \pi m k T}+p \tau_{0} \exp \left(E_{d} / k T\right)},
\end{aligned}
$$

where $p_{2}, m_{2}$ are the partial pressure and molecular mass of the vapor from the desired product, respectively. The relationship between the radius of spherical nanoparticles and their mass is

$$
M=\frac{4 \pi r^{3} m_{2}}{3 V_{s 0}} \quad \text { or } \quad \mathrm{d} M=\frac{4 \pi r^{2} m_{2}}{V_{s 0}} \mathrm{~d} r .
$$


In the surface constraint condition, the radius growth rate of nanoparticles is obtained by

$$
\frac{\mathrm{d} r}{\mathrm{~d} t}=\frac{\varphi_{m} V_{s 0} p_{2} \sqrt{m / m_{2}}}{\varphi_{m} \sqrt{2 \pi m k T}+p \tau_{0} \exp \left(E_{d} / k T\right)} .
$$

Thus, in the particle growth process restricted by the surface constraint condition, the radius growth rate of nanoparticles is proportional to the partial pressure of the vapor from the desired product. Thus, the radius growth rate remains constant at a constant pressure; that is, the particle radius increases linearly with time.

According to the results of the transfer constraint and surface constraint conditions, particulate growth is affected largely by assistant gases in the particulate growth zone. In the surrounding space, the assistant gas molecules collide with vapor molecules and delay their movement to the particulate surface. On the particulate surface, the assistant gas molecules occupy the adsorbing sites of the surface and prevent vapor molecules from condensing. Therefore, the increase of the assistant gas pressure will decrease the nanoparticle growth rate. However, this finding is only one aspect of the process. The following section will discuss the importance of other gases.

\section{Temperature Constraint Condition}

The heat transfer process that occurs on the particulate surface is another condition that restricts the nanoparticle growth process. In this condition, the primary issue is whether or not the latent phase change heat released by the adsorbed vapor molecules can be transported out of the particles in time. If the latent heat cannot be removed, the temperature of the particles will continue to increase, which will then increase the saturated vapor pressure of the particulate surface. When the saturated vapor pressure of the particulate surface is equivalent to the partial vapor pressure in the surrounding space, the molecular adsorption rate is similar to the molecular reevaporation rate on the particulate surface, which will then stop particulate growth. To ensure particulate growth, the heat removed by the desorbing gas molecules from the surface should be equal to the latent phase change heat released as a result of the condensation of the vapor molecules.

The heat dissipation process of nanoparticles according to molecular dynamics is as follows: the gas molecules, which come from the surrounding space that has a lower temperature, travel at a slow thermal motion velocity until they reach the particulate surface that has a higher temperature, achieve heat balance with the surface, escape from the surface at a fast thermal motion velocity, and then return to the surrounding space. The kinetic energy difference between the escaping velocity and the incident velocity of gas molecules is the heat quantity taken from the surface. The nanoparticle sizes during the growth process are close to the mean free path of gas molecules. Thus, the quantity of the dissipating heat can be calculated according to the free molecular flow.

Four gases are present in the region of the laser beam, namely, precursor gas, the vapor of the desired solid product, residual gas of reaction byproducts, and protective argon gas. All gases can participate in the heat transfer of nanoparticles; however, the latter two are particularly crucial. The heat transfer occurs under a free molecular flow state, and the action of each gas composition can be dealt with independently.

First, the heat exchange between a single gas composition and the particulate surface is considered. When the number density of gas molecules is $\mathrm{n}$, the velocity distribution of incident molecules per unit time per unit area at the particulate surface is [12]

$$
\mathrm{d} N=\frac{1}{4} n c \cdot f(c) \mathrm{d} c,
$$

where $c(\mathrm{~m} / \mathrm{s})$ is the molecular thermal velocity and $f(c)$ is the Maxwell velocity distribution function. The thermal motion of each molecule has an incident kinetic energy $(1 / 2) m c^{2}$. Thus, the total energy of the thermal motion introduced by the incident molecules to the per unit area surface in per unit time is

$$
\begin{aligned}
E_{g} & =\int_{0}^{\infty} \frac{1}{2} m c^{2} \cdot \frac{1}{4} n c \cdot f(c) \mathrm{d} c \\
& =p \sqrt{\frac{2 k T}{\pi m}} \quad\left(\mathrm{~J} \cdot \mathrm{m}^{-2} \cdot \mathrm{s}^{-1}\right) .
\end{aligned}
$$

According to Knudsen's law of reflection [12], the total thermal motion energy carried by the desorbing molecules from per unit area surface in per unit time can also be calculated by using (15), in which the only change is that temperature $T$ takes the value of particulate surface $T_{s}$. Therefore, the heat loss per unit area of the particulate surface in per unit time due to a kind of gas composition is

$$
Q_{i}=p_{i} \sqrt{\frac{2 k}{\pi m_{i}}}\left(\sqrt{T_{s}}-\sqrt{T_{g}}\right) \cdot \alpha_{i}\left(\mathrm{~J} \cdot \mathrm{m}^{-2} \cdot \mathrm{s}^{-1}\right) \quad(i=1,3,4),
$$

where $T_{s}$ and $T_{g}$ denote the respective temperatures of the surface and the gas, $p_{i}$ represents the partial pressure of the gases, and $\alpha_{i}$ is the accommodation coefficient that corresponds to the probability of the diffused reflection of gas molecules. The value of $\alpha_{i}$ is between 0 and 1 . Consider the following:

$$
\Delta H=p_{2} f h_{s 0} \sqrt{\frac{m_{2}}{2 \pi k T_{g}}} \quad\left(\mathrm{~J} \cdot \mathrm{m}^{-2} \cdot \mathrm{s}^{-1}\right),
$$

where $h_{s 0}\left(\mathrm{~J} \cdot \mathrm{kg}^{-1}\right)$ is the latent phase change heat (enthalpy) of per unit mass of vapor. The apparent value of $h_{s 0}$ on the nanoparticle surface is less than that on a macroscopically flat solid surface because of the size effect of the nanoparticles.

The heat loss quantity removed by using vapor molecular sublimation is

$$
Q_{2}=p_{2} \sqrt{\frac{2 k}{\pi m_{2}}}\left(\sqrt{T_{s}}-\sqrt{T_{g}}\right) \cdot \alpha_{2}(1-f) \quad\left(\mathrm{J} \cdot \mathrm{m}^{-2} \cdot \mathrm{s}^{-1}\right) .
$$


Thus, the condition that does not hinder the nanoparticle growth process can be shown as

$$
\Delta H \leq \sum_{i} Q_{i} \quad(i=1,2,3,4) .
$$

The mass growth rate determined by the temperature constraint condition is obtained by

$$
\frac{\mathrm{d} M}{\mathrm{~d} t}=4 \pi r^{2} \cdot \frac{p_{2}}{\sqrt{2 \pi m_{2} k T_{2}}} \cdot m_{2} f \leq \frac{4 \pi r^{2}}{h_{s 0}} \sum_{i} Q_{i} .
$$

By using (12), the radius growth rate of nanoparticles in the temperature constraint condition can be obtained as

$$
\frac{\mathrm{d} r}{\mathrm{~d} t} \leq \frac{V_{s 0}}{m h_{s 0}} \sum_{i} Q_{i}
$$

When the partial pressure and temperature of every composition are constant, the radius growth rate of nanoparticles determined by the temperature constraint condition is also constant; that is, the particle radii increase linearly with time, which is consistent with the particulate growth process under surface constraint conditions.

Finally, a specific case of particle growth under the temperature constraint condition is discussed. If the heat dissipation function of the precursor gas and protective argon gas is neglected and the condensation coefficient of vapor molecules on the particulate surface is assumed to be $f=1$, therefore, $Q_{1}=Q_{2}=Q_{4}=0$ is present in (19). The temperature constraint condition can then be simplified as

$$
p_{2} h_{s 0} \sqrt{\frac{m_{2}}{2 \pi k T_{g}}} \leq p_{3} \sqrt{\frac{2 k}{\pi m_{3}}}\left(\sqrt{T_{s}}-\sqrt{T_{g}}\right) ;
$$

that is,

$$
\frac{p_{2}}{p_{3}} \leq \frac{2 k}{h_{s 0} \sqrt{m_{2} m_{3}}}\left(\sqrt{T_{s} T_{g}}-T_{g}\right) .
$$

If the partial pressure ratio of the desired solid product vapor $(i=2)$ and reaction byproducts residual gas $(i=3)$ meets (23), the particle growth process will not be restricted by the heat transfer process. In some experiments that involve laser synthesis of nanopowders, the high usage of precursor material can be obtained when the assistant gas $\left(\mathrm{H}_{2}\right.$ or $\left.\mathrm{Ar}\right)$ is diluted by the precursor gas. This result occurs because both the reaction and the particulate growth are part of the temperature constraint process. The assistant gas can improve heat transfer ability and address heat transfer limitations. As a result, the condensation ratio of the vapor increases and a greater amount of nanopowders is obtained.

\section{Conclusions}

A molecular dynamics study was conducted to study the physical mechanism of the nanoparticle growth process in the laser synthesis of nanopowders. The particle growth process is analyzed in detail and is divided into five steps in which the microscopic heat and mass transfer process occurs either in the surrounding space or on the particulate surface. Three constraint conditions, namely, transfer constraint, surface constraint, and temperature constraint conditions, are proposed and modeled. The final diameter of the nanoparticles is restricted by the mass transfer in the surrounding space around the particles and calculated by using (7). The nanoparticle growth rate is affected by both mass and heat transfers on the particulate surface and is estimated by using (13) and (21), respectively.

\section{References}

[1] R. Fantoni, E. Borsella, S. Piccirillo, R. Ceccato, and S. Enzo, "Laser synthesis and crystallographic characterization of ultrafine SiC powders," Journal of Materials Research, vol. 5, no. 1, pp. 143-150, 1990.

[2] A. A. Francis and H. L. Marcus, "Laser synthesis of boron nitride powder," International Journal of Powder Metallurgy, vol. 37, no. 6, pp. 67-72, 2001.

[3] B. David, N. Pizúrová, O. Schneeweiss et al., "Magnetic properties of nanometric Fe-based particles obtained by laserdriven pyrolysis," Journal of Physics and Chemistry of Solids, vol. 68, no. 5-6, pp. 1152-1156, 2007.

[4] J. L. H. Chau, C. Y. Chen, M. C. Yang et al., "Femtosecond laser synthesis of bimetallic Pt-Au nanoparticles," Materials Letters, vol. 65, no. 4, pp. 804-807, 2011.

[5] Y. A. Kotov, O. M. Samatov, M. G. Ivanov et al., "Production and characteristics of composite nanopowders using a fiber ytterbium laser," Technical Physics, vol. 56, no. 5, pp. 652-655, 2011.

[6] R. Fantoni, L. De Dominicis, S. Martelli et al., "NIR and UV spectroscopic techniques as tools to control nanoparticles growth in laser pyrolisys process," in Proceedings of the Fiber Optic Sensor Technology and Applications 2001, vol. 4578 of Proceedings of SPIE, pp. 165-173, November 2002.

[7] Y. Liu, Y. Yin, J. Li, and G. Wang, "Design of LICVD equipment for preparation of nano powder and study on laser threshold," Chinese Optics Letters, vol. 2, no. 11, pp. 643-646, 2004.

[8] M. Amara and M. El Ganaoui, "Simulation of heat and mass transfers inside a nanopowders synthesis reactor," Progress in Computational Fluid Dynamics, vol. 6, no. 6, pp. 371-378, 2006.

[9] M. J. Šćepanović, M. Grujić-Brojčin, Z. D. Dohčević-Mitrović, and Z. V. Popović, "Temperature dependence of the lowest frequency Eg Raman mode in laser-synthesized anatase $\mathrm{TiO}_{2}$ nanopowder," Applied Physics A, vol. 86, no. 3, pp. 365-371, 2007.

[10] H. W. Emmons, "Fundamentals of gas dynamics" in The Condensation Phenomenon in High Speed Fluid, H. G. Stephen, Ed., Princeton University Press, 1958.

[11] P. Chen, Vacuum Technology Scientific Basis, National Defence Industry Press, Beijing, China, 1987.

[12] B. Gao and S. Cui, Vacuum Physics, Scicence Press, Beijing, China, 1983. 

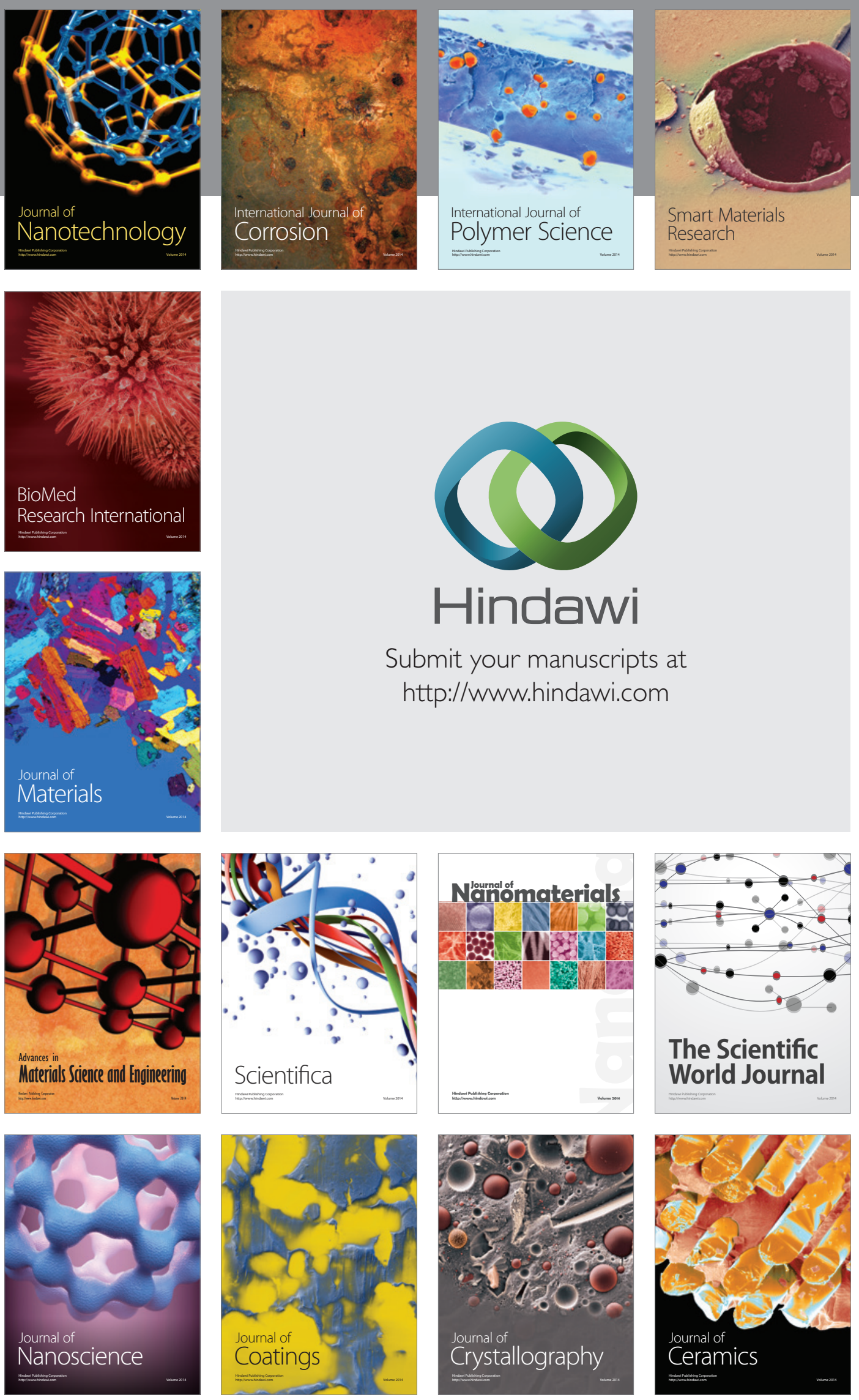

The Scientific World Journal

Submit your manuscripts at

http://www.hindawi.com

\section{World Journal}

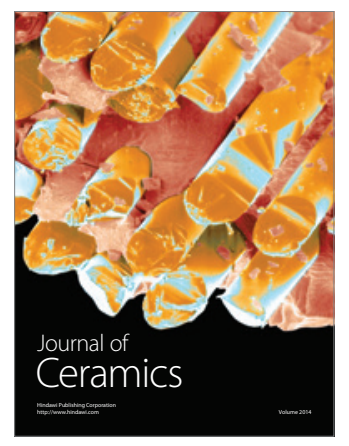

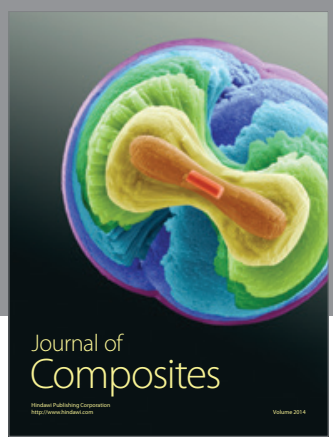
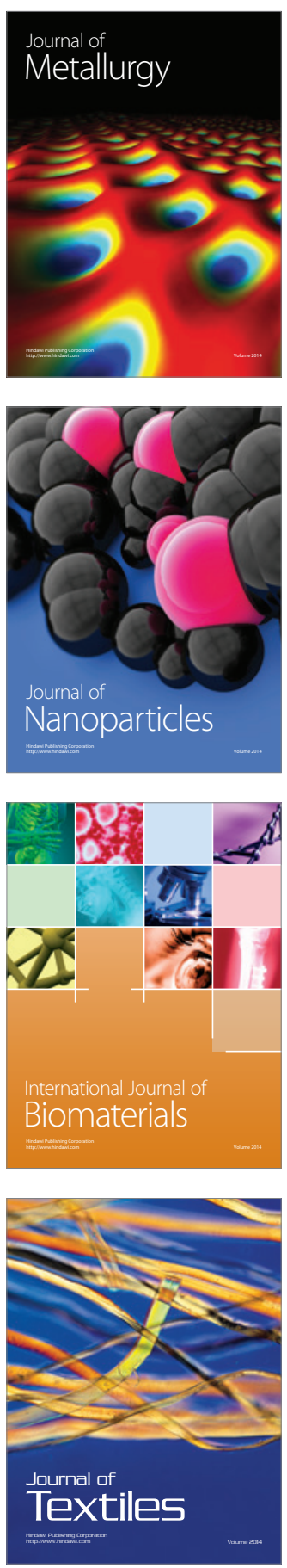